\title{
Mobile Ad Hoc Collaboration
}

\author{
Gerd Kortuem \\ Computer Science Dept. \\ University of Oregon \\ Eugene OR 97403 \\ $+15413464426$ \\ kortuem@cs.uoregon.edu
}

\author{
Hans-Werner Gellersen \\ Lancaster University \\ Computing Department \\ Lancaster, LA1 4YR, UK \\ +44(0) 1524593823 \\ hwg@comp.lancs.ac.uk
}

\author{
Mark Billinghurst \\ Human Interface Tech. Lab. \\ University of Washington \\ Seattle, WA 98195 \\ +1 2065435075 \\ grof@hitl.washington.edu
}

Keywords mobile collaboration, ad-hoc networks, CSCW, spontaneous collaboration, MANET, PAN

\section{INTRODUCTION}

The combination of personal mobile devices and mobile adhoc networks creates opportunities for new forms of mobile collaboration involving interaction between people who are co-located and organized in an unforeseeable ad hoc way. Possible application scenarios include informal social interactions in public places, opportunistic meetings in office settings, ad-hoc collaboration of emergency response teams and educational multi-user applications for use in classrooms.

The success of ad hoc networks may largely depend on the applications and social benefits they provide. Yet, prior research in this area has primarily focused on network technologies while mostly ignoring questions relating to the design, use and evaluations of such applications. Consequently, there is a general lack of understanding for how such networks can facilitate ad hoc collaboration.

\section{A RESEARCH FRAMEWORK}

To date, the bulk of CSCW research has investigated remote or face-to-face collaboration based on meeting places with dedicated infrastructure, such as meeting rooms, and for planned collaboration. However in everyday life social interaction is often driven by chance encounters of mobile people. Therefore the question how such interactions can be supported and enriched by mobile technology becomes an important research topic.

In order to systematically investigate social, usage and technical aspects of mobile ad hoc collaboration we must establish a comprehensive research framework that combines research in the following three areas:

1. Empirical research into the question of how and for what purposes people interact and collaborate when they are mobile. Past research has lead to the identification of several important forms of mobility and mobile interactions. For example, local mobility was identified as playing a major role even at work sites normally categorized as containing non-mobile work. Another

Copyright is held by the author/owner(s).

CHI 2002, April 20-25, 2002, Minneapolis, Minnesota, USA. ACM 1-58113-454-1/02/0004. important categorization distinguishes between planned meetings, opportunistic meetings and informal communication.

2. Experimental research aimed at creating prototype systems and applications.

3. Sociological research on the impact of mobile technology on the way people interact and collaborate. Not surprisingly, work in this category is sparse.

\section{WORKSHOP GOAL}

This workshop aims to provide a forum for the discussion of human-factors issues related to the design and evaluation of collaborative applications for mobile ad-hoc and personalarea networks. In particular, it tries to advance the understanding of how ad-hoc network applications can facilitate spontaneous collaboration.

The intention of this workshop it to bring together researchers and practitioners from a wide variety of disciplines such as CSCW, HCI, Ubiquitous Computing, Mobile Computing, Wearable Computing, Distributed Computing, and Wireless Networking to discuss issues related to the design, development, and evaluation of mobile ad hoc network applications. The immediate objective is to provide a forum for intellectual debate; the ultimate goal is to define a research agenda for the area as a whole and to stimulating new research initiatives. Among the issues the workshop will address are:

1. How can chance interactions be supported and enriched by current advances in mobile device and network technology?

2. What design methods lend themselves to the development of mobile ad hoc systems?

3. How can we evaluate systems for ad hoc collaboration?

The topics of interest include but are not limited to:

- Presence-based and face-to-face collaboration

- Opportunistic meetings and spontaneous collaboration

- Mobile and wearable communities

- Mobile groupware

- Design and evaluation methods

- Security and privacy aspects

- Usability aspects and social impacts

More information on the workshop is available at http://www.cs.uoregon.edu/research/wearables/chi2002ws 